\title{
ON THE MAXIMUM PARTIAL SUMS OF SEQUENCES OF INDEPENDENT RANDOM VARIABLES $\left({ }^{1}\right)$
}

\author{
BY \\ KAI LAI CHUNG
}

1. Introduction. In this paper we deal with a sequence of independent random variables $X_{n}, n=1,2, \cdots$. We write

$$
\begin{aligned}
S_{n} & =\sum_{\nu=1}^{n} X_{\nu}, \\
S_{n}^{*} & =\max _{1 \leqq \nu \leqq n}\left|S_{\nu}\right| .
\end{aligned}
$$

Two types of fundamental limit theorems are known about $S_{n}$, the one clustering around the central limit theorem and the other the law of the iterated logarithm.

In 1945 Feller [12](2) called attention to the study of the behavior of $S_{n}^{*}$. Since then an important result has been obtained by Erdös and Kac [8], namely, the limiting distribution of $S_{n}^{*}$ for sufficiently general sequences of $X_{n}$. This corresponds to the central limit theorem for $S_{n}$. Now under certain conditions when the distribution of $S_{n}$ tends to the normal distribution, an estimate of the difference of the two distributions has been given by Liapounoff [17], Cramér [5], Berry [3] and Essen [9]. Cramér [6] and Feller [10] have also obtained more precise estimates for this difference for certain domains of variation of $S_{n}$, which proved essential to the general form of the law of the iterated logarithm. It is therefore of interest to make the same kind of investigations regarding $S_{n}^{*}$. The problem is more difficult, since we have as yet no standard tools as in the case of $S_{n}$. We shall prove in this direction, as consequences of a more general but less handy inequality (Lemma 7), two theorems corresponding to the two types of estimation mentioned above. In order to state them we introduce the following notations. Let $E(X)$ denote the mathematical expectation of $X$. We shall assume that for each $X$, the first moment is zero, and the third absolute moment is finite. Thus we can write

$$
\begin{aligned}
& E\left(X_{\nu}\right)=0 ; \\
& E\left(X_{\nu}^{2}\right)=\sigma_{\nu}^{2} ; \quad s_{n}^{2}=\sum_{\nu=1}^{n} \sigma_{\nu}^{2} ;
\end{aligned}
$$

Presented to the Society, September 4, 1947; received by the editors May 27, 1947.

(1) The present paper is the revised form of a Dissertation for the degree of Doctor of Philosophy accepted by Princeton University, 1947.

(2) Numbers in brackets refer to the references cited at the end of the paper. 


$$
E\left(\left|X_{\nu}\right|^{3}\right)=\gamma_{\nu} ; \quad \Gamma_{n}=\sum_{\nu=1}^{n} \gamma_{\nu}
$$

Naturally we assume that $s_{n} \rightarrow \infty$. We shall further make the following assumption:

$$
\max _{1 \leqq \nu \leqq n} \gamma_{\nu} \sigma_{\nu}^{-2}=O\left(s_{n}^{1-\theta}\right)
$$

where $\theta$ is a fixed but arbitrarily small positive number. Then we can prove the following two theorems.

THEOREM 1. If $c$ is a positive constant, then we have

$$
\begin{aligned}
\operatorname{Pr}\left(S_{n}^{*}<c s_{n}\right)= & \frac{4}{\pi} \sum_{i=0}^{\infty} \frac{(-1)^{i}}{2 i+1} \exp \left(-\frac{(2 i+1)^{2} \pi^{2}}{8 c^{2}}\right) \\
& +O\left(\left(\frac{\lg _{2} s_{n}}{\lg s_{n}}\right)^{1 / 2}\right) .
\end{aligned}
$$

THEOREM 2. If $g_{n} \downarrow 0$ and

$$
g_{n}^{-1}=O\left(\left(\lg _{2} s_{n}\right)^{1 / 2}\right)
$$

then we have( $\left.{ }^{3}\right)$

$$
\operatorname{Pr}\left(S_{n}^{*}<g_{n} S_{n}\right)=(1+o(1)) \exp \left(-\frac{\pi^{2}}{8 g_{n}^{2}}\right) .
$$

Theorem 2 is one of a number of possible statements; we give prominence to it here because it furnishes the means of proving the next group of theorems which we now consider.

We might attempt to extend the law of the iterated logarithm to $S_{n}{ }^{*}$. This turns out to be illusory since the same law holds for $S_{n}^{*}$ as for $S_{n}$. More precisely, if $\phi_{n} \uparrow \infty$, we have always ("i. o." standing for "infinitely of ten")

$$
\operatorname{Pr}\left(S_{n}^{*}>\phi_{n} s_{n} \text { i. o. }\right)=\operatorname{Pr}\left(S_{n}>\phi_{n} s_{n} \text { i. o. }\right) \text {. }
$$

This is obvious since both $S_{n}{ }^{*}$ and $\phi_{n} s_{n}$ are monotone increasing functions of $n$. Hence in particular three of Feller's theorems [11] read as follows:

I. If $\sup \left|X_{n}\right|=O\left(s_{n}\left(\lg _{2} s_{n}\right)^{-3 / 2}\right)$ and $\phi_{n}^{2}=2 \quad \lg _{2} s_{n}+3 \quad \lg _{3} s_{n}+2 \quad \lg _{4} s_{n}$ $+\cdots+2 \lg _{p-1} s_{n}+(2+\delta) \lg _{p} s_{n}$ then the probability

$$
\operatorname{Pr}\left(S_{n}^{*}>s_{n} \phi_{n} \text { i. o. }\right)
$$

(8) Added in proof. For the application of Theorem 2 in Lemma 9 it is important to notice that the constant in the $o(1)$ term in (9) depends only on the constants in the $O(1)$ terms in (6) and (8), and the $\theta$ in (6), but otherwise is independent of the random variables considered. 
is equal to zero or one according as $\delta$ is positive or not.

II. If $\phi_{n} \uparrow \infty$ and

$$
\sup \left|X_{n}\right|=O\left(\frac{s_{n}}{\phi_{n}^{3}}\right)
$$

then (10) is equal to zero or one according as the series

$$
\sum_{n} \frac{\sigma_{n}^{2}}{s_{n}^{2}} \phi_{n} e^{-(1 / 2) \phi_{n}^{2}}
$$

is convergent or divergent.

III. If $\phi(t) \uparrow \infty$ and

$$
\sup \left|X_{n}\right|=O\left(\frac{s_{n}}{\phi^{3}\left(s_{n}^{2}\right)}\right),
$$

then $\operatorname{Pr}\left(S_{n}^{*}>s_{n} \phi\left(s_{n}^{2}\right)\right.$ i. o.) is equal to zero or one according as the integral

$$
\int^{\infty} \frac{1}{t} \phi(t) e^{-(1 / 2) \phi^{2}(t)} d t
$$

is convergent or divergent.

These results give very precise upper bounds for $S_{n}^{*}$, with probability one. The question naturally arises as to the precise lower bounds for $S_{n}^{*}$. (We may mention that the analogous problem for $S_{n}$ has been treated by Erdös and the author [4] and is radically different.) In this connection Erdös has communicated to the author the following result: there exist two constants $c_{2}>c_{1}>0$ such that

$$
\operatorname{Pr}\left(c_{1}<\lim \inf \frac{S_{n}^{*}}{s_{n}\left(\lg _{2} s_{n}\right)^{-1 / 2}}<c_{2}\right)=1 .
$$

His method, of an elementary nature, does not seem capable of a sharper result. Using Theorem 2 stated above we can easily prove that

$$
\operatorname{Pr}\left(\lim \inf \frac{S_{n}^{*}}{s_{n}\left(\lg _{2} s_{n}\right)^{-1 / 2}}=8^{-1 / 2} \pi\right)=1 .
$$

This corresponds to Khintchine-Kolmogoroff's original form of the law of the iterated logarithm ([14] and [16]). However, we can go much further and prove the following theorems which are the exact counterparts of Feller's theorems cited above.

THEOREM 3. Under the assumptions (3) to (6), if 


$$
\phi_{n}^{2}=\lg _{2} s_{n}+2 \lg _{3} s_{n}+\lg _{4} s_{n}+\cdots+\lg _{p-1} s_{n}+(1+\delta) \lg _{p} s_{n}
$$

then

$$
\operatorname{Pr}\left(S_{n}^{*}<8^{-1 / 2} \pi \phi_{n}^{-1} s_{n} \text { i. o. }\right)
$$

is equal to zero or one according as $\delta$ is positive or not.

THEOREM 4. Under the same assumptions, if $\phi_{n} \uparrow \infty$, then (12) is equal to zero or one according as the series

$$
\sum \frac{\sigma_{n}^{2}}{s_{n}^{2}} \phi_{n}^{2} e^{-\phi_{n}^{2}}
$$

is convergent or divergent.

Theorem 3 is a particular case of Theorem 4.

THEOREM 5. Under the same assumptions, if $\phi\left(s_{n}^{2}\right) \uparrow \infty$, then

$$
\operatorname{Pr}\left(S_{n}^{*}<8^{-1 / 2} \pi \phi^{-1}\left(s_{n}^{2}\right) s_{n} \text { i. o. }\right)
$$

is equal to zero or one according as the integral

$$
\int^{\infty} \frac{1}{t} \phi^{2}(t) e^{-\phi^{2}(t)} d t
$$

is convergent or divergent.

The similarity between these theorems and Feller's is indeed striking. It should however be noted that the condition (6) is not the best possible, although it is weaker than those considered by Cramér [5]. That condition (6) can be trivially weakened will be apparent from the proof. But no complete settlement of the question seems in sight.

We outline the methods of proof as follows. We approximate the distribution on $S_{n}^{*}$ by that of

$$
\max _{1 \leqq j \leqq k}\left|S_{n_{j}}\right|
$$

where $k$ is an integer to be determined later and $0<n_{1}<\cdots<n_{k}=n$ is a suitably chosen sequence such that $s_{n_{j}}^{2} \sim j k^{-1} s_{n}^{2}$.

In $\$ 2$ we study the approximate distribution of (16). It is found to approach that of a $k$-dimensional normal distribution with a remainder we shall estimate. The treatment in Lemma $2\left({ }^{4}\right)$, much to be preferred to the

(4) In the special case of equal components Bergström's result [2] seems to imply a better estimate than Lemma 2 , replacing the factor $4^{k}$ by a fixed power of $k$. The improvement however is annulled by Lemma 3. It becomes essential in the problem of $\max S_{\nu}$, without the absolute value. We shall consider this elsewhere. 
author's original proof using characteristic functions, is due to G. A. Hunt.

In $\$ 3$ we estimate the difference between the distribution of $S_{n}^{*}$ and that of (16). This is done by a substantial improvement of the method of Erdös and $\mathrm{Kac}(8)$, using sharper estimates resulting from the one-dimensional Berry-Esseen estimate. To obtain the approximate distribution of $S_{n}^{*}$ it remains to evaluate the $k$-dimensional normal distribution obtained in $\$ 2$. The problem appears to be one of multiple integrals but has not been worked out directly. Instead we use a quantitative refinement of the "invariance principle" of Erdös and Kac and study the simplest case of random walk. This latter problem, being almost classical, has been treated by many authors with different methods. However as we require not only the limiting distribution but also a remainder no reference seems available in the literature. We shall obtain the precise result by going back to a combinatorial formula due (apparently) to Bachelier [1]. After this we combine the results in $\$ \S 2$ and 3 to establish a theorem (Lemma 7) which includes Theorems 1 and 2 as particular cases.

In $\$ 4$ we prove Theorems 3,4 and 5 . The proof of these theorems depends essentially on Theorem 2, which plays the role here as the theorem of CramérFeller does in the case of Feller's theorems cited above. Several arguments of Feller's are also used and the author's indebtedness to his previous work is considerable.

The author wishes to express his gratitude to Professor Cramér for his warm encouragement and valuable counsel. To Dr. Erdös, whose first result actually started the investigation, the author owes many heartfelt thanks for his sustained interest. To Mr. Hunt, who is responsible not only for Lemma 2 but for many corrections on the original manuscript, the author's gratitude is equally great.

2. An approximation theorem for a certain multi-dimensional distribution. We shall use $A_{1}, A_{2}, \cdots$ to denote absolute constants.

Let $n_{1}<\cdots<n_{k}=n$ be a subsequence of $1, \cdots, n$ defined by the following:

$$
s_{n_{j}}^{2} \leqq j k^{-1} s_{n}^{2}<s_{n_{j}+1}^{2}, \quad j=1, \cdots, k .
$$

Then $\left(S_{n_{1}}, \cdots, S_{n_{j}}\right)$ is a random point in $j$-dimensional space. Let its distribution function be

$$
F_{j}\left(u_{1}, \cdots, u_{j}\right)=\operatorname{Pr}\left(S_{n_{1}} \leqq u_{1}, \cdots, S_{n_{j}} \leqq u_{j}\right) .
$$

Write also

$$
F_{j}^{*}(x)=\operatorname{Pr}\left(S_{n_{j}}-S_{n_{j-1}} \leqq x\right), \quad S_{n_{0}}=0 .
$$

We put

$$
B_{j}^{2}=s_{n_{j}}^{2}-s_{n_{j-1}}^{2}
$$




$$
M_{n}=\max _{1 \leqq \nu \leqq n} \gamma_{\nu} \vec{\sigma}_{\nu}^{-2}
$$

LEMMA 1. We have

$$
F_{j}^{*}(x)=\Phi_{j}^{*}(x)+R_{j}^{*}(x),
$$

where $\Phi_{j}^{*}(x)$ is the normal distribution function with mean 0 and variance $B_{j}^{2}$, and $\left|R_{j}^{*}(x)\right| \leqq A_{1} M_{n} B_{j}^{-1}$.

This is a restatement of the Berry-Esseen theorem.

LEMMA 2. Suppose that (6) holds and also that

$$
\max _{1 \leqq \nu \leqq n} \sigma_{\nu}^{2}=o\left(k^{-1} s_{n}\right) \text {. }
$$

Then we have

$$
\left|F_{j}\left(u_{1}, \cdots, u_{j}\right)-\Phi_{j}\left(u_{1}, \cdots, u_{j}\right)\right| \leqq A_{2} k^{1 / 2} 4^{i} M_{n} s_{n}^{-1},
$$

where $\Phi_{j}\left(u_{1}, \cdots, u_{j}\right)$ is the $j$-dimensional normal distribution function with the same moments of the first and second order as $F_{j}\left(u_{1}, \cdots, u_{j}\right)$.

Proof. From (17), (19) and (21) it is easy to see that

$$
B_{j} \sim k^{-\mathbf{1} / 2} s_{n}
$$

Hence by Lemma 1, we have

$$
\left|R_{j}^{*}(x)\right| \leqq A_{2} k^{1 / 2} M_{n} s_{n}^{-1} .
$$

For $j=1, R_{1}(x)=R_{1}^{*}(x)$; hence (22) is true for $j=1$. Now we use induction on $j$. Assume that

$$
\left|R_{j}\left(u_{1}, \cdots, u_{j}\right)\right| \leqq A_{2} k^{1 / 2} 4^{j} M_{n} s_{n}^{-i} .
$$

We have, by the definition (18),

$$
\begin{aligned}
F_{j+1}\left(u_{1}, \cdots, u_{j+1}\right)= & \int_{-\infty}^{\infty} F_{j}\left(u_{1}, \cdots, u_{j-1}, \min \left(u_{j}, u_{j+1}-x\right)\right) d F_{j+1}^{*}(x) \\
& =\int_{-\infty}^{\infty}\left\{\Phi_{j}\left(u_{1}, \cdots, u_{j-1}, \min \left(u_{j}, u_{j+1}-x\right)\right)\right. \\
\left.+R_{j}\left(u_{1}, \cdots, u_{j-1}, \min \left(u_{j}, u_{j+1}-x\right)\right)\right\} d\left[\Phi_{j+1}^{*}(x)+R_{j+1}^{*}(x)\right] & \\
= & \Phi_{j+1}\left(u_{1}, \cdots, u_{j+1}\right)+\int R_{j} d \Phi_{j+1}^{*} \\
& +\int R_{j} d \Phi_{j+1}^{*}+\int \Phi_{j} d R_{j+1}^{*} .
\end{aligned}
$$


Evidently we have

$$
\begin{aligned}
& \left|\int R_{j} d \Phi_{i+1}^{*}\right| \leqq \sup \left|R_{j}\right| \\
& \left|\int R_{j} d R_{j+1}^{*}\right| \leqq 2 \sup \left|R_{j}\right| .
\end{aligned}
$$

Finally, using integration by parts, we have

$$
\begin{aligned}
& \int_{-\infty}^{\infty} \Phi_{j}\left(u_{1}, \cdots, u_{j-1}, \min \left(u_{j}, u_{j+1}-x\right)\right) d R_{j+1}^{*}(x) \\
&=\int_{-\infty}^{u_{j+1}-u_{j}} \Phi_{j}\left(u_{1}, \cdots, u_{j}\right) d R_{j+1}^{*}(x) \\
& \quad+\int_{u_{j+1}-u_{j}}^{\infty} \Phi_{j}\left(u_{1}, \cdots, u_{j}, u_{j+1}-x\right) d R_{j+1}^{*}(x) \\
&=\Phi_{j}\left(u_{1}, \cdots, u_{j}\right) R_{j+1}^{*}\left(u_{j+1}-u_{j}\right)-\Phi_{j}\left(u_{1}, \cdots, u_{j}\right) R_{j+1}^{*}\left(u_{j+1}-u_{j}\right) \\
&+\int_{u_{j+1}-u_{j}}^{\infty} R_{j+1}^{*}(x) d \Phi_{j}\left(u_{1}, \cdots, u_{j-1}, u_{j+1}-x\right) .
\end{aligned}
$$

Hence the absolute value of the left-hand side is less than or equal to

$$
\sup \left|R_{j+1}^{*}\right| \text {. }
$$

Substituting these estimates into (22), we obtain

$$
\left|F_{j+1}-\Phi_{j+1}\right| \leqq 3\left(\sup \left|R_{j}\right|+\sup \left|R_{j+1}^{*}\right|\right) .
$$

From (25) and (26), we have

$$
\begin{aligned}
\left|F_{j+1}-\Phi_{j+1}\right| & \leqq 3 A_{2} k^{1 / 2} M_{n} s_{n}^{-1}\left(4^{j}+1\right) \\
& \leqq A_{2} 4^{j+1} k^{1 / 2} M_{n} s_{n}^{-1}
\end{aligned}
$$

Thus the induction is complete.

Now we put, for non-negative $u_{j}^{\prime}$ 's,

$$
\begin{aligned}
& F_{0}\left(u_{1}, \cdots, u_{k}\right)= \operatorname{Pr}\left(\left|S_{n_{1}}\right| \leqq s_{n} u_{1}, \cdots,\left|S_{n_{k}}\right| \leqq s_{n} u_{k}\right) \\
& \Phi_{0}\left(u_{1}, \cdots, u_{k}\right)= \frac{s_{n}^{k}}{(2 \pi)^{k / 2} B_{1} \cdots B_{k}} \int_{-u_{1}}^{u_{1}} \cdots \int_{-u_{k}}^{u_{k}} \\
& \cdot \exp \left(-\frac{1}{2} \sum_{j=1}^{k} \frac{s_{n}^{2}}{B_{j}^{2}}\left(x_{j}-x_{j-1}\right)^{2}\right) d x_{1} \cdots d x_{k} .
\end{aligned}
$$

LEMMA 3. Under the same assumptions as in Lemma 2, we have 


$$
\left|F_{0}-\Phi_{0}\right| \leqq A_{3}(10)^{k} M_{n} s_{n}^{-1} \text {. }
$$

Proof. Taking $j=k$ in (22), we have

$$
\left|F_{k}-\Phi_{k}\right| \leqq A_{2} k^{1 / 2} 4^{k} M_{n} s_{n}^{-1} \leqq A_{3} 5^{k} M_{n} s_{n}^{-1} .
$$

It is well known that we have

$$
\begin{aligned}
F_{0}\left(u_{1}, \cdots, u_{k}\right)= & F_{k}\left(s_{n} u_{1}, \cdots, s_{n} u_{k}\right) \\
& -F_{k}\left(-s_{n} u_{1}, s_{n} u_{2}, \cdots, s_{n} u_{k}\right)-\cdots \\
& -F_{k}\left(s_{n} u_{1}, \cdots, s_{n} u_{k-1},-s_{n} u_{k}\right) \\
& +F_{k}\left(-s_{n} u_{1},-s_{n} u_{2}, s_{n} u_{3}, \cdots, s_{n} u_{k}\right)+\cdots \\
& +(-1)^{k} F_{k}\left(-s_{n} u_{1}, \cdots,-s_{n} u_{k}\right)
\end{aligned}
$$

and a similar relation holds between $\Phi_{k}$ and $\Phi_{0}$. Since there are $2^{k}$ terms on the right-hand side, (29) follows immediately from (30). It is not hard to obtain the explicit form of $\Phi_{0}\left(u_{1}, \cdots, u_{k}\right)$ in (28) by considering the covariance matrix.

3. The distribution of the maximum partial sum. Let $c$ be a positive constant; $g_{n}$ a monotone function of $n ; \epsilon_{n}=o(1)$.

LemMa 4. Suppose that (6) and (21) are satisfied, and also that we have

$$
\begin{aligned}
\epsilon_{n}^{2} g_{n}^{2} & =o\left(k^{-3 / 2} s_{n}^{\theta}\right), \\
\sigma_{n}^{2} & =o\left(\left(\epsilon_{n}^{2} g_{n}^{2} s_{n}^{3-\theta}\right)^{2 / 3}\right) .
\end{aligned}
$$

Then we have

$$
\operatorname{Pr}\left(S_{n}^{*}<c g_{n} s_{n}\right) \geqq \operatorname{Pr}\left(\max _{1 \leqq j \leqq k}\left|S_{n_{j}}\right|<\left(c-\epsilon_{n}\right) \dot{g}_{n} s_{n}\right)-R_{n}
$$

where

$$
R_{n} \leqq A_{4}\left(k^{-1 / 2} \epsilon_{n}^{-1} g_{n}^{-1} \exp \left(-4^{-12} \epsilon_{n}^{2} g_{n}^{2} k\right)+\left(\epsilon_{n} g_{n} s_{n}^{\theta}\right)^{-2 / 3}\right) .
$$

Proof. Write

$$
\begin{aligned}
P_{n} & =\operatorname{Pr}\left(S_{n}^{*}<c g_{n} s_{n}\right), \\
W_{r} & =\operatorname{Pr}\left(S_{r-1}^{*}<c g_{n} s_{n},\left|S_{r}\right| \geqq c g_{n} s_{n}\right) .
\end{aligned}
$$

Then we have

$$
\sum_{r=1}^{n} W_{r}=\operatorname{Pr}\left(S_{n}^{*} \geqq c g_{n} s_{n}\right)=1-P_{n} \leqq 1 .
$$

Suppose that $n_{j}<\gamma \leqq n_{j+1}$. We have 


$$
\begin{aligned}
W_{r}= & \operatorname{Pr}\left(S_{r-1}^{*}<c g_{n} s_{n},\left|S_{r}\right| \geqq c g_{n} s_{n}\right) \operatorname{Pr}\left(\left|S_{n_{j+1}}-S_{r}\right| \geqq \epsilon_{n} g_{n} s_{n}\right) \\
& +\operatorname{Pr}\left(S_{r-1}^{*}<c g_{n} s_{n},\left|S_{r}\right| \geqq c g_{n} s_{n},\left|S_{n_{j+1}}-S_{r}\right|<\epsilon_{n} g_{n} s_{n}\right) .
\end{aligned}
$$

Let $A>0$ be an integer to be determined later. If $n_{j+1}-r \leqq A$, we have by the Tchebychef inequality,

$$
\operatorname{Pr}\left(\left|S_{n_{j+1}}-S_{r}\right| \geqq \epsilon_{n} g_{n} s_{n}\right) \leqq\left(s_{n_{j+1}}^{2}-s_{n_{j+1}-A+1}^{2}\right)\left(\epsilon_{n} g_{n} s_{n}\right)^{-2} .
$$

If $n_{j+1}-r=B>A$, we have by the Berry-Esseen theorem,

$$
\operatorname{Pr}\left(\left|S_{n_{j+1}}-S_{r}\right| \geqq \epsilon_{n} g_{n} s_{n}\right)=\left(\frac{2}{\pi}\right)^{1 / 2} \int_{v}^{\infty} e^{-u^{2} / 2} d u+O(\rho)
$$

where

$$
v=\epsilon_{n} g_{n} s_{n}\left(s_{n_{j+1}}^{2}-s_{n_{j+1}-A+1}^{2}\right)^{-1 / 2}
$$

and

$$
\rho=M_{n}\left(\sum_{\nu=n_{j+1}-A+1}^{n_{j+1}} \sigma_{\nu}^{2}\right)^{-1 / 2} .
$$

Hence from (38), since $A<B \leqq n_{j+1}-n_{j}, s_{n_{j+1}}^{2}-s_{n_{j+1}-A+1}^{2} \leqq s_{n_{j+1}}^{2}-s_{n_{j}}^{2}=B_{j+1}^{2}$,

$$
\operatorname{Pr}\left(\left|S_{n_{j+1}}-S_{r}\right| \geqq \epsilon_{n} g_{n} s_{n}\right)
$$

$$
\leqq A_{5}\left(\frac{B_{j+1}}{\epsilon_{n} g_{n} s_{n}} \exp \left(-\frac{\epsilon_{n}^{2} g_{n}^{2} s_{n}^{2}}{2 B_{j+1}^{2}}\right)+\frac{M_{n}}{\left(s_{n_{j+1}}^{2}-s_{n_{j+1}-A+1}^{2}\right)^{1 / 2}}\right) .
$$

We choose $A$ such that

$$
\frac{M_{n}}{\left(s_{n_{j+1}}^{2}-s_{n_{j+1}-A+1}\right)^{1 / 2}} \sim \frac{s_{n_{j+1}}^{2}-s_{n_{j+1}-A+1}^{2}}{\epsilon_{n}^{2} g_{n}^{2} s_{n}^{2}}
$$

that is,

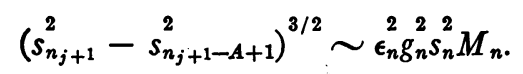

Since $n_{j+1}-A+1 \geqq n_{j}$ this is possible if, for example,

$$
\underset{\epsilon_{n}}{2} g_{n}^{2} s_{n}^{2} M_{n}=o\left(B_{j+1}^{3}\right)=o\left(k^{-3 / 2} s_{n}^{3}\right)
$$

by (23), and also if

$$
\sigma_{n}^{2}=o\left(\left(\epsilon_{n}^{2} g_{n}^{2} s_{n}^{2} M_{n}\right)^{2 / 3}\right) .
$$

These are implied by the conditions (31) and (32), on account of (6). Hence we obtain from (37) and (39) 


$$
\operatorname{Pr}\left(\left|S_{n j+1}-S_{r}\right| \geqq \epsilon_{n} g_{n} s_{n}\right) \leqq A_{5}\left(\frac{B_{j+1}}{\epsilon_{n} g_{n} s_{n}} \exp \left(-\frac{\epsilon_{n}^{2} g_{n}^{2} s_{n}^{2}}{2 B_{j+1}^{2}}\right)+\left(\epsilon_{n} g_{n} s_{n}^{0}\right)^{-2 / 3}\right) .
$$

Since $B_{j+1}^{2} s_{n}^{-2} \sim k^{-1}$ by (23), we obtain

$$
\operatorname{Pr}\left(\left|S_{n_{j+1}}-S_{r}\right| \geqq \epsilon_{n} g_{n} s_{n}\right) \leqq A_{4}\left(\frac{1}{k^{1 / 2} \epsilon_{n} g_{n}} \exp \left(-\frac{\epsilon_{n}^{2} g_{n}^{2} k}{4}\right)+\left(\epsilon_{n} g_{n} s_{n}^{0}\right)^{-2 / 3}\right) .
$$

If we denote the maximum of the left-hand side of this inequality for all $r$ by $R_{n}$, (36) becomes

$$
W_{r} \leqq R_{n}+\operatorname{Pr}\left(S_{r-1}^{*}<c g_{n} s_{n},\left|S_{r}\right| \geqq c g_{n} s_{n},\left|S_{n_{j+1}}-S_{r}\right|<\epsilon_{n} g_{n} s_{n}\right) .
$$

From (35) and (40), we obtain

$\operatorname{Pr}\left(S_{n}^{*} \geqq c g_{n} s_{n}\right)$

$$
\begin{aligned}
& \leqq R_{n}+\sum_{j=0}^{k-1} \sum_{r=n_{j+1}}^{n_{j+1}} \operatorname{Pr}\left(S_{r-1}^{*}<c g_{n} s_{n},\left|S_{r}\right| \geqq c g_{n} s_{n},\left|S_{n_{j+1}}-S_{r}\right|<\epsilon_{n} g_{n} s_{n}\right) \\
& \leqq R_{n}+\operatorname{Pr}\left(\max _{1 \leqq j \leqq k}\left|S_{n_{j}}\right| \geqq\left(c-\epsilon_{n}\right) g_{n} s_{n}\right) .
\end{aligned}
$$

This is equivalent to (33).

If in the function $F_{0}\left(u_{1}, \cdots, u_{k}\right)$ of (27) all the arguments are equal to $u$ we shall use the shorter notation $F_{0 k}(u)$; similarly for $\Phi_{0 k}$.

LEMma 5. Suppose that the condition (6) is satisfied, and also for $a \Theta>0$ we have

$$
\frac{8 \lg 10 \cdot \Theta}{\theta} \frac{\lg _{2} s_{n}}{\lg s_{n}} \leqq \underset{\epsilon_{n} g_{n}^{2}}{2}=o\left(\frac{\dot{s}_{n}}{\left(\lg s_{n}\right)^{3 / 2}}\right) .
$$

Then if we choose

$$
k \sim \frac{\theta \lg s_{n}}{2 \lg 10}
$$

we have

$$
\Phi_{0 k}\left(\left(c-\epsilon_{n}\right) g_{n}\right)-L_{n} \leqq \operatorname{Pr}\left(S_{n}^{*}<c g_{n} s_{n}\right) \leqq \Phi_{0 k}\left(c g_{n}\right)+L_{n}
$$

where

$$
L_{n}=O\left(\left(\lg s_{n}\right)^{-\theta}\right)
$$

Proof. From (6) it follows that 


$$
\sigma_{n} \leqq \gamma_{n} \sigma_{n}^{-2} \equiv O\left(s_{n}^{1-\theta}\right) .
$$

Hence with the $k$ in (42) condition (21) is satisfied. Further condition (32) in Lemma 4 is satisfied with $\epsilon_{n}^{2} g_{n}^{2}$ satisfying (41). Condition (31) is clearly satisfied with (41) and the choice of $k$ in (42). Hence both Lemma 3 and Lemma 4 are applicable. Taking all the $u$ 's in (29) to be $\left(c-\epsilon_{n}\right) g_{n}$ and recalling (27) we obtain

$$
\begin{aligned}
\operatorname{Pr}\left(S_{n}^{*}<c g_{n} s_{n}\right) & \geqq F_{0 k}\left(\left(c-\epsilon_{n}\right) g_{n}\right)-R_{n} \\
& \geqq \Phi_{0 k}\left(\left(c-\epsilon_{n}\right) g_{n}\right)-A_{3}(10)^{k} s_{n}^{-1} M_{n}-R_{n} .
\end{aligned}
$$

On the other hand we have

$$
\operatorname{Pr}\left(S_{n}^{*}<c g_{n} s_{n}\right) \leqq F_{0 k}\left(c g_{n}\right) \leqq \Phi_{0 k}\left(c g_{n}\right)+A_{3}(10)^{k} s_{n}^{-1} M_{n} .
$$

We find from (42) and (46)

$$
\begin{gathered}
(10)^{k}=O\left(s_{n}^{\theta / 2}\right), \quad(10)^{k} s_{n}^{-1} M_{n}=O\left(s_{n}^{-\theta / 2}\right), \\
k^{-1 / 2} \epsilon_{n}^{-1} g_{n}^{-1} \exp \left(-4^{-1} \epsilon_{n}^{2} g_{n}^{2} k\right)=O\left(\left(\lg s_{n}\right)^{-\theta}\right), \\
\left(\epsilon_{n} g_{n} s_{n}^{-0 / 3}=O\left(s_{n}^{-\theta / 2}\right) .\right.
\end{gathered}
$$

Hence if we take

$$
L_{n}=R_{n}+A_{3}(10)^{k} s_{n}^{-1} M_{n}=O\left(\left(\lg s_{n}\right)^{-\Theta}\right)
$$

(45) and (46) imply (43).

LeMma 6. Suppose that for each $\nu$,

$$
X_{\nu}= \begin{cases}+1 & \text { with probability } 1 / 2 \\ -1 & \text { with probability } 1 / 2\end{cases}
$$

Then if $g_{n}=o\left(n^{1 / 2}\right)$, we have

$$
\operatorname{Pr}\left(S_{n}^{*}<c g_{n} n^{1 / 2}\right)=T\left(c g_{n}\right)+O\left(g_{n}^{-1} n^{-1 / 2}\right)+O\left(n^{-1 / 2}\right)
$$

where $T(x)$ is the distribution function defined by

$$
T(x)=\frac{4}{\pi} \sum_{i=0}^{\infty} \frac{(-1)^{i}}{2 i+1} \exp \left(-\frac{(2 i+1)^{2} \pi^{2}}{8 x^{2}}\right), \quad x>0 .
$$

Proof. Write, for integral $a$ and $b$,

$$
P(a)=\operatorname{Pr}\left(S_{n}=a,-b<S_{\nu}<b, \text { for } 0<\nu \leqq n\right) .
$$

By a formula due to Bachelier [1, pp. 252-253], 


$$
\begin{aligned}
2^{n} P(a)=C_{n,(n+a) / 2} & +\sum_{1 \leqq i \leqq(n+a) / 2 b}(-1)^{i} C_{n,(n+a) / 2-i b} \\
& +\sum_{1 \leqq i \leqq(n-a) / 2 b}(-1)^{i} C_{n,(n-a) / 2-i b}
\end{aligned}
$$

if $n$ and $a$ have the same parity, otherwise $P(a)=0$. Without loss of generality we may assume $n$ to be even, $b$ odd. Then

$$
\sum_{-b<a<b, a \equiv 0(\bmod 2)} C_{n,(n+a) / 2-i b}=\sum_{(-b+1) / 2 \leqq j \leqq(b-1) / 2} C_{n, n / 2+j-i b .}
$$

Write

$$
\begin{aligned}
P_{i}=P_{-i} & =\sum_{(-b+1) / 2 \leqq j \leqq(b-1) / 2} C_{n, n / 2+j-i b} \frac{1}{2^{n}} \\
& =\sum_{n / 2+1 / 2+\left(n^{1 / 2} / 2\right) \zeta_{1 i} \leqq m \leqq n / 2-1 / 2+\left(n^{1 / 2} / 2\right) \zeta_{2 i}} C_{n, m} ;
\end{aligned}
$$

where

$$
\zeta_{1 i}=-(2 i+1) b n^{-1 / 2}, \quad \zeta_{2 i}=-(2 i-1) b n^{-1 / 2} .
$$

Finally we write

$$
P=\sum_{-b<a<b} P(a)
$$

From a formula of Uspensky [16, p. 129], noticing that the limits of the range of $m$ are integers, we deduce easily that

$$
\begin{aligned}
\sum_{n / 2+1 / 2+\left(n^{1 / 2} / 2\right) \zeta 1 \leqq m \leqq n / 2-1 / 2+\left(n^{1 / 2} / 2\right) \zeta_{2}} C_{n, m} & \frac{1}{2^{n}} \\
= & \left(\frac{1}{2 \pi}\right)^{1 / 2} \int_{\zeta_{1}}^{\zeta_{2}} e^{-u^{2} / 2} d u+O\left(\frac{1}{n}\right) .
\end{aligned}
$$

Hence

$$
P_{-i}+P_{i}=\left(\frac{2}{\pi}\right)^{1 / 2} \int_{\zeta_{1 i}}^{\zeta_{2 i}} e^{-u^{2} / 2} d u+O\left(\frac{1}{n}\right) .
$$

Since $-b<a<b$,

$$
\begin{aligned}
& \left.\frac{1}{2^{n}}\right|_{1 \leqq i \leqq(n+a) / 2 b}(-1)^{i} C_{n,(n+a) / 2-i b}+\sum_{1 \leqq i \leqq(n-a) / 2 b}(-1)^{i} C_{n,(n-a) / 2-i b} \\
& \quad-\quad-\sum_{1 \leqq i \leqq n / 2 b}(-1)^{i}\left[C_{n,(n+a) / 2-i b}+C_{n,(n-a) / 2-i b}\right] \mid \leqq \frac{1}{2^{n}} .
\end{aligned}
$$

Hence 


$$
\left|\sum_{-b<a<b, a \equiv 0(\bmod 2)} P(a)-\sum_{1 \leqq i \leqq n / 2 b}(-1)^{i}\left(P_{i}+P_{-i}\right)\right|
$$

$$
\leqq \sum_{-b<a<b, a \equiv 0(\bmod 2)} \frac{1}{2^{n}} \leqq \frac{b}{2^{n}} .
$$

Therefore from (50) to (52),

$$
\begin{aligned}
P= & \left(\frac{1}{2 \pi}\right)^{1 / 2} \int_{\zeta_{10}}^{\zeta_{20}} e^{-u^{2} / 2} d u+O\left(\frac{1}{n}\right) \\
& +\sum_{1 \leqq i \leqq n / 2 b}(-1)^{i}\left\{\left(\frac{1}{2 \pi}\right)^{1 / 2} \int_{\zeta_{1 i}}^{\zeta_{2 i}} e^{-u^{2} / 2} d u+O\left(\frac{1}{n}\right)\right\}+O\left(\frac{b}{2^{n}}\right) \\
= & \left(\frac{1}{2 \pi}\right)^{1 / 2} \int_{\zeta_{10}}^{\zeta_{20}} e^{-u^{2} / 2} d u+\sum_{1 \leqq i \leqq n / 2 b}(-1)^{i}\left(\frac{2}{\pi}\right)^{1 / 2} \int_{\zeta_{1 i}}^{\zeta_{2 i}} e^{-u^{2} / 2} d u \\
& +O\left(\frac{1}{n}\right)+O\left(\frac{1}{b}\right)+O\left(\frac{b}{2^{n}}\right) .
\end{aligned}
$$

Since the terms are alternating in sign and decreasing in absolute value, we have

$$
\begin{aligned}
\left|\sum_{i>n / 2 b}(-1)^{i}\left(\frac{2}{\pi}\right)^{1 / 2} \int_{\zeta_{1 i}}^{\zeta_{2 i}} e^{-u^{2} / 2} d u\right| & \\
& \leqq\left(\frac{2}{\pi}\right)^{1 / 2} \int_{(n-b) n-1 / 2}^{(n+b) n-1 / 2} e^{-u^{2} / 2} d u=O\left(e^{-n / 3}\right),
\end{aligned}
$$

if $b=o(n)$.

Hence if $b=o(n)$, we obtain from (53) and (54),

$$
\begin{aligned}
P & =\left(\frac{1}{2 \pi}\right)^{1 / 2} \int_{\zeta_{10}}^{\zeta_{20}} e^{-u^{2} / 2} d u+\sum_{i=1}^{\infty}(-1)^{i}\left(\frac{2}{\pi}\right)^{1 / 2} \int_{\zeta_{1 i}}^{\zeta_{2 i}} e^{-u^{2} / 2} d u+O\left(\frac{1}{b}\right) \\
& =\left(\frac{1}{2 \pi}\right)^{1 / 2} \sum_{i=-\infty}^{\infty}(-1)^{i} \int_{(2 i-1) b n-1 / 2}^{(2 i+1) b n-1 / 2} e^{-u^{2} / 2} d u+O\left(\frac{1}{b}\right) \ldots
\end{aligned}
$$

We shall now construct a function $h(x)$ with period $2 \alpha$ as follows:

$$
\begin{aligned}
& h(x)=\left\{\begin{array}{rll}
1 & \text { if } & 0<x<\alpha / 2, \\
-1 & \text { if } & \alpha / 2<x<\alpha
\end{array}\right. \\
& h(x)=h(-x) ; \because h(x)=h(x+2 \alpha) \text {. }
\end{aligned}
$$

It is easy to find that

$$
h(x)=\frac{4}{\pi} \sum_{i=0}^{\infty} \frac{(-1)^{i}}{2 i+1} \cos \left(\frac{2 i+1}{\alpha} \pi x\right) .
$$


Taking $\alpha$ to be $2 b n^{-1 / 2}$ in the above, we have

$$
\begin{gathered}
\left(\frac{1}{2 \pi}\right)^{1 / 2} \sum_{i=-\infty}^{\infty}(-1)^{i} \int_{(2 i-1) b n-1 / 2}^{(2 i+1) b n-1 / 2} e^{-u^{2} / 2} d u=\left(\frac{1}{2 \pi}\right)^{1 / 2} \int_{-\infty}^{\infty} h(x) e^{-x^{2} / 2} d x \\
=\frac{4}{\pi} \sum_{i=0}^{\infty} \frac{(-1)^{i}}{2 i+1}\left(\frac{1}{2 \pi}\right)^{1 / 2} \int_{-\infty}^{\infty} e^{-x^{2} / 2} \cos \left(\frac{(2 i+1) \pi n^{1 / 2}}{2 b} x\right) d x \\
=\frac{4}{\pi} \sum_{i=0}^{\infty} \frac{(-1)^{i}}{2 i+1} \exp \left(-\frac{(2 i+1)^{2} \pi^{2} n}{8 b^{2}}\right)
\end{gathered}
$$

since

$$
\left(\frac{1}{2 \pi}\right)^{1 / 2} \int_{-\infty}^{\infty} e^{-x^{2} / 2} \cos t x d x=e^{-t^{2} / 2}
$$

Therefore from (55) and (56) we obtain

$$
P=T\left(b n^{-1 / 2}\right)+O\left(b^{-1}\right)
$$

Since by assumption $g_{n}=o\left(n^{1 / 2}\right), c g_{n} n^{1 / 2}=o(n)$; taking $b$ successively to be the nearest odd integers to $c g_{n} n^{1 / 2}$ in (54) and observing that $T\left(b n^{-1 / 2}\right)$ $-T\left(\operatorname{cg}_{n}\right)=O\left(n^{-1 / 2}\right)$ we obtain (48).

LEMMA 7. Returning to the general case, we have, if (6) and (41) are satisfied,

$$
T\left(\left(c-\epsilon_{n}\right) g_{n}\right)-H_{n} \leqq \operatorname{Pr}\left(S_{n}^{*}<c g_{n} s_{n}\right) \leqq T\left(\left(c+\epsilon_{n}\right) g_{n}\right)+H_{n}
$$

where $T(x)$ is defined in (49) and

$$
H_{n}=O\left(\left(\lg s_{n}\right)^{-\oplus}+g_{n}^{-1} s_{n}^{-1}\right) \text {. }
$$

Proof. For the special case (47), we have according to the general notation (4) and (5),

$$
\sigma_{m}^{2}=1, \quad \gamma_{m}=1, \quad s_{m}^{2}=m, \quad M_{m}=1 .
$$

Condition (6) is satisfied with $\theta=1$. Hence by Lemma 5 , if

$$
\frac{8 \lg 10 \cdot \Theta \lg _{2} m^{1 / 2}}{\theta \lg m^{1 / 2}} \leqq{\epsilon_{m}^{\prime 2} g_{m}^{\prime 2}}^{\prime 2}=o\left(\frac{m^{1 / 2}}{(\lg m)^{3 / 2}}\right)
$$

we have from (43),

$$
\Phi_{1 k}\left(\left(c-\epsilon_{m}^{\prime}\right) g_{m}^{\prime}\right)-L_{m} \leqq \operatorname{Pr}\left(S_{m}^{*}<c g_{m}^{\prime} m^{1 / 2}\right) \leqq \Phi_{1 k}\left(c g_{m}^{\prime}\right)+L_{m},
$$

where, by (42), $k$ is given by

$$
k \sim \frac{\theta \lg m}{4 \lg 10}
$$


and where $\Phi_{1 k}$ is obtained from $\Phi_{0 k}$ in (28) after we replace $s_{n}^{2}$ by $m$ and $B_{j}$ by $B_{j}^{\prime}$ defined according to (17) and (19) by

$$
m_{j} \leqq j k^{-1} m<m_{j}+1, B_{j}^{\prime 2}=m_{j}-m_{j-1},
$$

and where

$$
L_{m}=O\left((\lg m)^{-\theta}\right) .
$$

On the other hand, by Lemma 6, we have for the special case in question

$$
\operatorname{Pr}\left(S_{m}^{*}<c g_{m}^{\prime} m^{1 / 2}\right)=T\left(c g_{m}^{\prime}\right)+O\left(g_{m}^{\prime-1} m^{-1 / 2}\right)+O\left(m^{-1 / 2}\right) .
$$

Substituting from (58) into (60) we obtain

$$
\Phi_{1 k}\left(c g_{m}^{\prime}\right)-L_{m} \leqq T\left(c g_{m}^{\prime}\right)+O\left(g_{m}^{\prime-1} m^{-1 / 2}+m^{-1 / 2}\right) \leqq \Phi_{1 k}\left(c g_{m}^{\prime}\right)+L_{m}
$$

From (62) we deduce

$$
\begin{array}{r}
\Phi_{1 k}\left(\left(c-\epsilon_{m}^{\prime}\right) g_{m}^{\prime}\right) \geqq T\left(\left(c-\epsilon_{m}^{\prime}\right) g_{m}^{\prime}\right)+O\left(g_{m}^{\prime-1} m^{-1 / 2}+m^{-1 / 2}\right)+O\left(L_{m}\right) \\
\Phi_{1 k}\left(c g_{m}^{\prime}\right) \leqq T\left(\left(c+\epsilon_{m}^{\prime}\right) g_{m}^{\prime}\right)+O\left(g_{m}^{\prime-1} m^{-1 / 2}+m^{-1 / 2}\right)+O\left(L_{m}\right)
\end{array}
$$

Now putting

$$
m=\left[s_{n}^{2}\right], \quad \epsilon_{m}^{\prime}=\epsilon_{n}, \quad g_{m}^{\prime}=g_{n},
$$

we obtain from (63) and (64) the following: if

$$
\frac{8 \lg 10 \cdot \Theta}{\theta} \frac{\lg _{2} s_{n}}{\lg s_{n}} \leqq \underset{\epsilon_{n}^{2} g_{n}^{2}}{2}=o\left(\frac{s_{n}^{\theta}}{\left(\lg s_{n}\right)^{3 / 2}}\right)
$$

then we have

$$
\begin{aligned}
T\left(\left(c-\epsilon_{n}\right) g_{n}\right)-K_{n} & \leqq \Phi_{1 k}\left(\left(c-\epsilon_{n}\right) g_{n}\right) \leqq \Phi_{1 k}\left(c g_{n}\right) \\
& \leqq T\left(\left(c+\epsilon_{n}\right) g_{n}\right)+K_{n},
\end{aligned}
$$

where

(42 bis)

$$
\begin{aligned}
k & \sim \frac{\theta \lg s_{n}}{2 \lg 10}, \\
K_{n} & =O\left(\left(\lg s_{n}\right)^{-\theta}+g_{n}^{-1} s_{n}^{-1}\right) .
\end{aligned}
$$

Writing $\lambda_{j}=s_{n} B_{j}^{-1}, \lambda_{j}^{\prime}=\left[s_{n}^{2}\right]^{1 / 2} B_{j}^{\prime-1}$ we have from (28)

$$
\begin{aligned}
\Phi_{0}\left(u_{1}, \cdots, u_{k}\right) & \\
& =\frac{\lambda_{1} \cdots \lambda_{k}}{(2 \pi)^{k / 2}} \int_{-u_{1}}^{u_{1}} \cdots \int_{-u_{k}}^{u_{k}} \exp \left(-\frac{1}{2} \sum_{j=1}^{k} \lambda_{j}^{2}\left(x_{j}-x_{j+1}\right)^{2}\right) d x_{1} \cdots d x_{k} .
\end{aligned}
$$


It is easy to verify that

$$
\left|\frac{\partial \Phi_{0}}{\partial \lambda_{j}}\right| \leqq \frac{3}{2 \lambda_{j}} .
$$

Hence

$$
\left|\Phi_{0 k}-\Phi_{1 k}\right| \leqq \frac{3}{2} \sum_{j=1}^{k} \frac{1}{\lambda_{j}}\left|\lambda_{j}-\lambda_{j}^{\prime}\right| .
$$

Since $\sigma_{n}=O\left(s_{n}^{1-\theta}\right), B_{j}^{2}=s_{n}^{2} k^{-1}+O\left(s_{n}^{2-2 \theta}\right)$,

$$
\lambda_{j}^{2}=\frac{s_{n}^{2}}{B_{j}^{2}}=k\left(1+O\left(\frac{k}{s_{n}^{2 \theta}}\right)\right) .
$$

The same holds for $\lambda_{j}^{\prime 2}$. Thus $\left|\lambda_{j}-\lambda_{j}^{\prime}\right|=O\left(k^{2} s_{n}^{-2 \theta}\right)$; and by (42 bis) and (67),

$$
\left|\Phi_{0 k}-\Phi_{1 k}\right|=O\left(s_{n}^{-\theta}\right) \text {. }
$$

Therefore from (65) we obtain

$$
\begin{aligned}
T\left(\left(c-\epsilon_{n}\right) g_{n}\right)-J_{n} & \leqq \Phi_{0 k}\left(\left(c-\epsilon_{n}\right) g_{n}\right) \leqq \Phi_{0 k}\left(c g_{n}\right) \\
. & \leqq T\left(\left(c+\epsilon_{n}\right) g_{n}\right)+J_{n},
\end{aligned}
$$

where $k$ is given by (42 bis) and from (66) and (68) we have

$$
J_{n}=O\left(R_{n}\right)+O\left(s_{n}^{-\theta}\right)=O\left(\left(\lg s_{n}\right)^{-\theta}+g_{n}^{-1} s_{n}^{-1}\right) .
$$

Using (69) in (43), Lemma 5, we obtain

$$
T\left(\left(c-\epsilon_{n}\right) g_{n}\right)-H_{n} \leqq \operatorname{Pr}\left(S_{n}^{*}<c g_{n} s_{n}\right) \leqq T\left(\left(c+\epsilon_{n}\right) g_{n}\right)+H_{n}
$$

where $H_{n}=O\left(J_{n}\right)+O\left(L_{n}\right)$. Hence by (44) and (70) we have established (58) and (59).

Proof of Theorem 1. Taking $g_{n}=1$ in Lemma 5, we get

$$
T\left(c-\epsilon_{n}\right)-H_{n} \leqq \operatorname{Pr}\left(S_{n}^{*}<c s_{n}\right) \leqq T\left(c+\epsilon_{n}\right)+H_{n},
$$

where

$$
H_{n}=O\left(\left(\lg s_{n}\right)^{-\Theta}\right) \text {. }
$$

Now we have, by the mean-value theorem,

$$
\begin{array}{r}
\exp \left(\frac{-(2 i+1)^{2} \pi^{2}}{8\left(c+\epsilon_{n}\right)^{2}}\right)-\exp \left(\frac{-(2 i+1)^{2} \pi^{2}}{8 c^{2}}\right) \\
\quad \leqq \frac{(2 i+1)^{2} \pi^{2}}{4 c^{3}} \exp \left(-\frac{(2 i+1)^{2} \pi^{2}}{8\left(c+\epsilon_{n}\right)^{2}}\right) \epsilon_{n} .
\end{array}
$$


Hence

$$
T\left(c+\epsilon_{n}\right)-T(c) \leqq \frac{4}{\pi} \sum_{i=0}^{\infty} \frac{(2 i+1) \pi^{2}}{4 c^{3}} \exp \left(-\frac{(2 i+1)^{2} \pi^{2}}{8\left(c+\epsilon_{n}\right)^{2}}\right) \epsilon_{n}=O\left(\epsilon_{n}\right) .
$$

Thus (71) becomes

$$
\operatorname{Pr}\left(S_{n}^{*}<c s_{n}\right)=T(c)+O\left(\epsilon_{n}\right)+O\left(\left(\lg s_{n}\right)^{-\Theta}\right) .
$$

Choosing, for example, $\Theta=1$ and

$$
\epsilon_{n}^{2}=\frac{8 \lg 10}{\theta} \frac{\lg _{2} s_{n}}{\lg s_{n}}
$$

which is permissible by (41), we obtain (7) from (72).

Proof of Theorem 2. We have

$$
\frac{4}{\pi} \exp \left(-\frac{\pi^{2}}{8 x^{2}}\right)-\frac{4}{3 \pi} \exp \left(-\frac{9 \pi^{2}}{8 x^{2}}\right) \leqq T(x) \leqq \frac{4}{\pi} \exp \left(-\frac{\pi^{2}}{8 x^{2}}\right) .
$$

Since $\epsilon_{n} \downarrow 0$, we have if $\epsilon_{n}<4^{-1}$,

$$
\begin{aligned}
T\left(\left(1+\epsilon_{n}\right) g_{n}\right) & \leqq \frac{4}{\pi} \exp \left(-\frac{\pi^{2}}{8 g_{n}^{2}}\left(1+\epsilon_{n}\right)^{-2}\right) \\
& \leqq \frac{4}{\pi} \exp \left(-\frac{\pi^{2}}{8 g_{n}^{2}}\left(1-2 \epsilon_{n}\right)\right) \\
& =\frac{4}{\pi} \exp \left(-\frac{\pi^{2}}{8 g_{n}^{2}}\right) \exp \left(\frac{\pi^{2} \epsilon_{n}}{4 g_{n}^{2}}\right) \\
T\left(\left(1-\epsilon_{n}\right) g_{n}\right) & \geqq \frac{4}{\pi} \exp \left(-\frac{\pi^{2}}{8 g_{n}^{2}}\left(1-\epsilon_{n}\right)^{-2}\right)-\frac{4}{3 \pi} \exp \left(-\frac{9 \pi^{2}}{8 g_{n}^{2}}\right) \\
& \geqq \frac{4}{\pi} \exp \left(-\frac{\pi^{2}}{8 g_{n}^{2}}\left(1+4 \epsilon_{n}\right)\right)-\frac{4}{3 \pi} \exp \left(-\frac{9 \pi^{2}}{8 g_{n}^{2}}\right) \\
& \geqq \frac{4}{\pi} \exp \left(-\frac{\pi^{2}}{8 g_{n}^{2}}\right) \exp \left(-\frac{\pi^{2} \epsilon_{n}}{2 g_{n}^{2}}\right)-\frac{4}{3 \pi} \exp \left(-\frac{9 \pi^{2}}{8 g_{n}^{2}}\right) .
\end{aligned}
$$

Choosing

$$
\epsilon_{n}^{2}=\frac{8 \lg 10 \cdot \Theta}{\theta} \frac{\lg _{2} s_{n}}{g_{n}^{2} \lg s_{n}}
$$

then (41) is satisfied, and from (8),

$$
\frac{\epsilon_{n}^{2}}{g_{n}^{4}}=\frac{8 \lg 10 \cdot \Theta}{\theta} \frac{\lg _{2} s_{n}}{g_{n}^{6} \lg s_{n}}=o(1)
$$


Hence we have

$$
\begin{array}{r}
\exp \left(\frac{\pi^{2} \epsilon_{n}}{4 g_{n}^{2}}\right)=1+o(1), \\
\exp \left(-\frac{\pi^{2} \epsilon_{n}}{2 g_{n}^{2}}\right)=1+o(1) .
\end{array}
$$

Since $g_{n} \downarrow 0$, we have

$$
\exp \left(-\frac{9 \pi^{2}}{8 g_{n}^{2}}\right)=o\left(\exp \left(-\frac{\pi^{2}}{8 g_{n}^{2}}\right)\right)
$$

Thus from (73) and (74), we obtain

$$
\begin{aligned}
\frac{4}{\pi}(1+o(1)) \exp \left(-\frac{\pi^{2}}{8 g_{n}^{2}}\right) & \leqq T\left(\left(1-\epsilon_{n}\right) g_{n}\right) \leqq T\left(\left(1+\epsilon_{n}\right) g_{n}\right) \\
& \leqq \frac{4}{\pi}(1+o(1)) \exp \left(-\frac{\pi^{2}}{8 g_{n}^{2}}\right)
\end{aligned}
$$

Therefore (58) becomes

$$
\operatorname{Pr}\left(S_{n}^{*}<g_{n} s_{n}\right)=\frac{4}{\pi}(1+o(1)) \exp \left(-\frac{\pi^{2}}{8 g_{n}^{2}}\right)+O\left(\left(\lg s_{n}\right)^{-\Theta}\right) .
$$

Since we may choose $\Theta$ arbitrarily large, (9) follows on account of (8).

4. Some strong limit theorems. Since we shall deal with indices $n, \nu, k$ and so on, which ultimately tend to infinity, we shall of ten omit mention of this proviso. Thus, sometimes our statements are true only when the appropriate index is sufficiently large.

The condition (6) is assumed in this section. From (6) it follows:

$$
\sigma_{n}=O\left(s_{n}^{1-\theta}\right) \text {, }
$$

Let $\psi_{n} \uparrow \infty$, and

$$
\psi_{n}=O\left(\left(\lg _{2} s_{n}\right)^{1 / 2}\right) .
$$

Taking $g_{n}=\psi_{n}^{-1}$ in Theorem 2 , we have

$$
A_{6} e^{-\psi_{n}^{2}} \leqq \operatorname{Pr}\left(S_{n}^{*}<8^{-1 / 2} \pi s_{n} \psi_{n}^{-1}\right) \leqq A_{7} e^{-\psi_{n}^{2}}
$$

We shall construct a subsequence $\left\{n_{k}\right\}, k=1,2, \cdots$, as follows. Take $a>0$. Put $n_{1}=1$. Suppose that $n_{k}$ is defined already, then since $s_{n} \uparrow$, there is a unique $n_{k+1}$ such that

$$
s_{n_{k+1}-1} \leqq s_{n_{k}}\left(1+a \psi_{n_{k}}^{-2}\right) \leqq s_{n_{k+1}}
$$


Hence (for $k$ sufficiently large)

$$
s_{n_{k+1}-1}^{2} \leqq s_{n_{k}}^{2}\left(1+3 a \psi_{n_{k}}^{-2}\right) .
$$

By virtue of (75) and (76), we have

$$
\begin{aligned}
& s_{n_{k+1}}^{2} \leqq s_{n_{k}}^{2}+3 a s_{n_{k}}^{2} \psi_{n_{k}}^{-2}+\sigma_{n_{k+1}}^{2} \leqq s_{n_{k}}^{2}+4 a s_{n_{k+1}}^{2} \psi_{n_{k}}^{-2} ; \\
& s_{n_{k+1}}^{2} \leqq s_{n_{k}}^{2}\left(1-4 a \psi_{n_{k}}^{-2}\right)^{-1} .
\end{aligned}
$$

Thus there exists $b>a$ such that

$$
s_{n_{k}}\left(1+a \psi_{n_{k}}^{-2}\right) \leqq s_{n_{k+1}} \leqq s_{n_{k}}\left(1+b \psi_{n_{k}}^{-2}\right)
$$

For simplicity we shall write $k^{\prime}$ for $n_{k}, s_{k}^{\prime}$ for $s_{n_{k}}, \psi_{k}^{\prime}$ for $\psi_{n_{k}}$, and so on.

LemMa 8. Suppose that $\psi_{n} \uparrow \infty$ and (76) holds. Let $\left\{n_{k}\right\}$ be any sequence satisfying (77). Then if

$$
\sum_{k} e^{-\psi_{n_{k}}^{2}}<\infty
$$

we have

$$
\operatorname{Pr}\left(S_{n}^{*}<8^{-1 / 2} \pi s_{n} \psi_{n}^{-1} \text { i. o. }\right)=0 .
$$

Proof. From ( 9 bis) we have

$$
\operatorname{Pr}\left(S_{k^{\prime}}^{*}<\frac{\pi}{8^{1 / 2}} \frac{s_{k}^{\prime}}{\left(\psi_{k}^{\prime 2}-3 b\right)^{1 / 2}}\right) \leqq A_{7} e^{-\left(\psi_{k}^{\prime 2}-3 b\right)}
$$

Hence by (78)

$$
\sum_{k=1}^{\infty} \operatorname{Pr}\left(S_{k^{\prime}}^{*}<\frac{\pi}{8^{1 / 2}} \frac{s_{k}^{\prime}}{\left(\psi_{k}^{\prime 2}-3 b\right)^{1 / 2}}\right)<\infty .
$$

By the lemma of Borel-Cantelli (see, for example [13, pp. 26-27]), we conclude that

$$
\operatorname{Pr}\left(S_{k^{\prime}}^{*}<\frac{\pi}{8^{1 / 2}} \frac{s_{k}^{\prime}}{\left(\psi_{k}^{\prime 2}-3 b\right)^{1 / 2}} \text { i. o. }\right)=0
$$

that is,

(80) $\quad \operatorname{Pr}\left(S_{k^{\prime}}^{*} \geqq \frac{\pi}{8^{1 / 2}} \frac{s_{k}^{\prime}}{\left(\psi_{k}^{\prime 2}-3 b\right)^{1 / 2}}\right.$ for all sufficiently large $\left.k\right)=1$.

Now suppose that $n_{k}<n \leqq n_{k+1}$. Then if

$$
S_{k^{\prime}}^{*} \geqq \frac{\pi}{8^{1 / 2}} \frac{s_{k}^{\prime}}{\left(\psi_{k}^{\prime 2}-3 b\right)^{1 / 2}}
$$


we have by (77)

$$
\begin{aligned}
S_{n}^{*} & \geqq S_{k^{\prime}}^{*} \geqq \frac{\pi}{8^{1 / 2}} \frac{s_{k+1}^{\prime}}{\left(\psi_{k}^{\prime 2}-3 b\right)^{1 / 2}} \frac{s_{k}^{\prime}}{s_{k+1}^{\prime}} \\
& \geqq \frac{\pi}{8^{1 / 2}} s_{k+1}^{\prime}\left(\psi_{k}^{\prime 2}-3 b\right)^{-1 / 2}\left(1+b \psi_{k}^{\prime-2}\right)^{-1} .
\end{aligned}
$$

If $k$ is sufficiently large, we have

$$
S_{n}^{*} \geqq \frac{\pi}{8^{1 / 2}} \frac{s_{k+1}^{\prime}}{\psi_{k}^{\prime}} \geqq \frac{\pi}{8^{1 / 2}} \frac{s_{n}}{\psi_{n}} .
$$

Thus (80) entails

$$
\operatorname{Pr}\left(S_{n}^{*}>\frac{\pi}{8^{1 / 2}} \frac{S_{n}}{\psi_{n}} \text { for all sufficiently large } n\right)=1 .
$$

This is equivalent to (79).

LEMMA 9. Suppose that $\psi_{n} \uparrow \infty$ and (76) holds. Let $\left\{n_{k}\right\}$ be any sequence satisfying (77). Then if

$$
\sum_{k} e^{-\psi_{n_{k}}^{2}}=\infty
$$

we have

$$
\operatorname{Pr}\left(S_{n}^{*}<\frac{\pi}{8^{1 / 2}} \frac{s_{n}}{\psi_{n}} \text { i. o. }\right)=1 .
$$

Proof. By (77), given $s_{\boldsymbol{k}_{\nu-1}}^{\prime}$, there is a unique $\nu$ such that

$$
s_{k_{\nu}}^{\prime} \leqq s_{k_{\nu-1}}^{\prime} \psi_{k_{\nu-1}}^{\prime 3}<s_{k_{\nu}+1}^{\prime}
$$

From ( 9 bis) we have, if $c>1 / 8$ is any constant,

$$
\operatorname{Pr}\left(S_{k^{\prime}}^{*}<\frac{\pi}{8^{1 / 2}} \frac{s_{k}^{\prime}}{\left(\psi_{k}^{\prime 2}+8 C\right)^{1 / 2}}\right) \geqq A_{6} e^{-\left(\psi_{k}^{\prime 2}+8 C\right)} .
$$

Hence by (81) we have

$$
\sum_{\nu=1}^{\infty} \sum_{k=k_{\nu}}^{k_{v+1}^{-1}} \operatorname{Pr}\left(S_{k^{\prime}}^{*}<\frac{\pi}{8^{1 / 2}} \frac{s_{k}^{\prime}}{\left(\psi_{k}^{\prime 2}+8 C\right)^{1 / 2}}\right)=\infty .
$$

Let $\{\nu(r)\}, r=1,2, \cdots$, denote the subsequence of $\nu=1,2, \cdots$ for which

$$
\psi^{2}\left(k_{\nu(r)+1}^{\prime}\right)>\psi^{2}\left(k_{\nu(r)-1}^{\prime}\right)+1
$$

Then we have 


$$
\operatorname{Pr}\left(S_{k^{\prime}}^{*}<\frac{\pi}{8^{1 / 2}} \frac{s_{k}^{\prime}}{\left(\psi_{k}^{\prime}+8 C\right)^{1 / 2}}\right) \leqq A_{7} e^{-\left(\psi_{k}^{\prime 2}+8 C\right)} \leqq A_{8} \psi^{-2}\left(k^{\prime}\right) e^{-\psi^{2}\left(k^{\prime}\right) / 2}
$$

From (83),

$$
\psi_{k_{\nu}}^{\prime 3} \geqq \frac{s_{k_{\nu+1}}^{\prime}}{s_{k_{\nu}}^{\prime}} \geqq \prod_{k=k_{\nu}}^{k_{\nu+1}-1}\left(1+\frac{a}{\psi^{\prime 2}}\right) \geqq a \sum_{k=k_{\nu}}^{k_{\nu+1}-1} \frac{1}{\psi^{\prime 2}}
$$

Hence

$$
\begin{aligned}
\sum_{k=k_{\nu(r)}}^{k_{\nu(r)+1-1}} \operatorname{Pr}\left(S_{k^{\prime}}^{*}<\frac{\pi}{8^{1 / 2}} \frac{s_{k}}{\left(\psi_{k}^{\prime}+8 C\right)^{1 / 2}}\right) & \leqq A_{8} e^{-\psi^{2}\left(k_{\nu(r)}^{\prime}\right) / 2} \sum_{k=k_{\nu(r)}}^{k_{\nu(r)+1-1}} \overline{\psi_{k}^{\prime 2}} \\
& \leqq \frac{A_{8}}{a} \psi_{k_{\nu(r)}^{\prime 3}}^{\prime 3} e^{-\psi^{2}\left(k_{\nu(r)) / 2}^{\prime}\right.} \leqq A_{9} e^{-\psi^{2}\left(k_{\nu(r)}^{\prime}\right) / 4}
\end{aligned}
$$

Thus by (85)

$$
\sum_{r} \sum_{k=k_{\nu(r)}}^{k_{\nu(r)+1-1}} \operatorname{Pr}\left(S_{k^{\prime}}^{*}<\frac{\pi}{8^{1 / 2}} \frac{s_{k}^{\prime}}{\left(\psi_{k}^{\prime 2}+8 C\right)^{1 / 2}}\right) \leqq A_{9} \sum_{r} e^{-\psi^{2}\left(k_{\nu(r)}^{\prime}\right) / 4}<\infty .
$$

From (82) and (86) we conclude that if we delete the values of $\nu$ equal to $\nu(r), r=1,2, \cdots$, in (84) the remaining series is still divergent. Without loss of generality we may then assume that, for some fixed $\nu_{0}$,

$$
\sum_{\nu=1, \nu \equiv \nu_{0}(\bmod 2)}^{\infty} \sum_{k=k_{\nu}}^{k_{\nu+1}-1} \operatorname{Pr}\left(S_{k^{\prime}}^{*}<\frac{\pi}{8^{1 / 2}} \frac{s_{k}^{\prime}}{\left(\psi_{k}^{\prime 2}+8 C\right)^{1 / 2}}\right)=\infty,
$$

where the prime after the summation indicates the omission of the values $\nu(r)$.

Denote by:

$E_{\mu}$ the event

$$
S_{\mu}^{*}<\frac{\pi}{8^{1 / 2}} \frac{s_{\mu}}{\psi_{\mu}}
$$

$E_{\nu-1}^{\prime}$ the event

$$
S_{k_{\nu-1}^{\prime}}^{*} \leqq C s_{k_{\nu-1}^{\prime}}
$$

$E_{\nu-1, \mu}$ the event

$$
\max _{k_{\nu-1}^{\prime}<\rho \leqq \mu}\left|S_{\rho}-S_{k_{\nu-1}^{\prime}}\right|<\frac{\pi}{8^{1 / 2}} \frac{s_{\mu}}{\left(\psi_{\mu}^{2}+8 C\right)^{1 / 2}}, \quad k_{\nu}^{\prime} \leqq \mu \leqq k_{\nu+1}^{\prime} .
$$

If $\nu \neq \nu(r)$, then from (83) and (77) we have, since $\psi_{k_{\nu}}^{\prime 2} \leqq \psi_{k_{\nu-1}}^{\prime 2}+1, \psi_{k_{\nu}}^{\prime 3}$ $\leqq 2^{1 / 2} \psi_{k_{\nu-1}}^{\prime 3}$ for lárge $\nu$,

$$
s_{k_{\nu-1}}^{\prime}<\psi_{k_{\nu-1}}^{\prime-3} s_{k_{\nu}}^{\prime}\left(1+b \psi_{k_{\nu}}^{\prime-2}\right) \leqq s_{k_{\nu}}^{\prime} 2^{1 / 2} \psi_{k_{\nu}}^{\prime-3}\left(1+b \psi_{k_{\nu}}^{\prime-2}\right) \leqq 2 s_{k_{\nu}}^{\prime} \psi_{k_{\nu}}^{\prime-3} .
$$


Then if we have the conjunction $E_{\nu-1}^{\prime} E_{\nu-1, \mu}$, we have by (88)

$$
\begin{aligned}
S_{\mu}^{*} & <\frac{\pi}{8^{1 / 2}} \frac{s_{\mu}}{\left(\psi_{\mu}^{2}+8 C\right)^{1 / 2}}+C s_{k_{\nu-1}}^{\prime}<\frac{\pi}{8^{1 / 2}}\left(\frac{s_{\mu}}{\left(\psi_{\mu}^{2}+8 C\right)^{1 / 2}}+\frac{2 C s_{\mu}}{\psi_{\mu}^{3}}\right) \\
& <\frac{\pi}{8^{1 / 2}} \frac{s_{\mu}}{\psi_{\mu}}\left(\frac{1}{\left(1+8 C \psi_{\mu}^{-2}\right)^{1 / 2}}+\frac{2 C}{\psi_{\mu}^{2}}\right)<\frac{\pi}{8^{1 / 2}} \frac{s_{\mu}}{\psi_{\mu}} .
\end{aligned}
$$

Therefore if $\nu \neq \nu(r)$, the conjunction $E_{\nu-1}^{\prime} E_{\nu-1, \mu}$ implies $E_{\mu}$. Writing

$$
F_{\nu}=\sum_{\mu=k_{\nu}^{\prime}}^{k_{\nu+1}^{\prime}-1} E_{\mu}, \quad F_{\nu}^{\prime}=\sum_{\mu=k_{\nu}^{\prime}}^{k_{\nu+1}^{\prime}-1} E_{\nu-1, \mu}
$$

we have, if $\nu \neq \nu(r), E_{\nu-1}^{\prime} F_{\nu}^{\prime}$ implies $F_{\nu}$, hence

$$
\begin{gathered}
\sum_{\nu=\nu_{1}}^{\infty} E_{\nu-1}^{\prime} F_{\nu}^{\prime} \text { implies } \sum_{\nu=\nu 1}^{\infty} F_{\nu}, \\
\operatorname{Pr}\left(\sum_{\nu=\nu_{1}}^{\infty} E_{\nu-1}^{\prime} F_{\nu}^{\prime}\right) \leqq \operatorname{Pr}\left(\sum_{\nu=\nu_{1}}^{\infty} F_{\nu}\right) .
\end{gathered}
$$

The events $F_{\nu}^{\prime}, F_{\nu+2}^{\prime}, F_{\nu+4}^{\prime}, \cdots$ are independent and $F_{j}^{\prime}$ for $j \geqq \nu$ is independent of $E_{\nu-1}^{\prime}$. By the Kolmogoroff inequality [15] we have

$$
\operatorname{Pr}\left(E_{\nu-1}^{\prime}\right) \geqq 1-1 / C^{2} \text {. }
$$

We obtain, by an obvious argument and (90), for all $\nu_{1} \geqq \nu_{0}$,

$$
\begin{aligned}
\operatorname{Pr}\left(\sum_{\nu=\nu_{1}}^{\infty} E_{\nu-1}^{\prime} F_{\nu}^{\prime}\right) & =\operatorname{Pr}\left(\sum_{\nu=\nu_{1}}^{\infty}\left(E_{\nu-1}^{\prime} F_{\nu}^{\prime}-E_{\nu-1}^{\prime} F_{\nu}^{\prime} \sum_{j=\nu+1}^{\infty} E_{j-1}^{\prime} F_{j}^{\prime}\right)\right. \\
& \geqq \operatorname{Pr}\left(\sum_{\nu=\nu_{1}}^{\infty} E_{\nu-1}^{\prime}\left(F_{\nu}^{\prime}-F_{\nu}^{\prime} \sum_{j=\nu+1}^{\infty} F_{j}^{\prime}\right)\right) \\
& =\sum_{\nu=\nu 1}^{\infty} \operatorname{Pr}\left(E_{\nu-1}^{\prime}\right) \operatorname{Pr}\left(F_{\nu}^{\prime}-F_{\nu}^{\prime} \sum_{j=\nu+1}^{\infty} F_{j}^{\prime}\right) \\
& \geqq\left(1-\frac{1}{C^{2}}\right) \operatorname{Pr}\left(\sum_{\nu=\nu_{1}}^{\infty} F_{\nu}^{\prime}\right) .
\end{aligned}
$$

Hence by (89) and (91) we have

$$
\begin{aligned}
\operatorname{Pr}\left(\sum_{\nu=\nu_{1}}^{\infty} F_{\nu}\right) & \geqq\left(1-\frac{1}{C^{2}}\right) \operatorname{Pr}\left(\sum_{i=\nu_{1}}^{\infty} F_{\nu}^{\prime}\right) \\
& \geqq\left(1-\frac{1}{C^{2}}\right) \operatorname{Pr}\left(\sum_{\nu=p_{1}, \nu \equiv \nu_{0}(\bmod 2)}^{\infty} F_{\nu}^{\prime}\right) .
\end{aligned}
$$

Since the events $F_{\nu_{0}}^{\prime}, F_{\nu_{0}+2}^{\prime}, F_{\nu_{0}+4}^{\prime}, \cdots$ are independent, by the lemma of 
Borel-Cantelli we know that

$$
\operatorname{Pr}\left(\sum_{\nu=\nu_{1}, \nu \equiv \nu_{0}(\bmod 2)}^{\infty} F_{\nu}^{\prime}\right)=1
$$

if and only if

$$
\sum_{\nu=1, \nu \equiv \nu_{0}(\bmod 2)}^{\infty} \operatorname{Pr}\left(F_{v}^{\prime}\right)=\infty .
$$

If we can prove (93), then from this remark and (91) we shall have for all $\nu_{1} \geqq \nu_{0}$, hence in fact for all $\nu_{1}$,

$$
\operatorname{Pr}\left(\sum_{\nu=\nu_{1}}^{\infty} F_{\nu}\right) \geqq 1-\frac{1}{C^{2}},
$$

a fortiori, for all $n_{1}$,

$$
\operatorname{Pr}\left(\sum_{n=n_{1}}^{\infty} E_{n}\right) \geqq 1-\frac{1}{C^{2}} .
$$

Since we may choose $C$ arbitrarily large while the left-hand side does not depend on $C$ we shall have proved for all $n_{1}, \operatorname{Pr}\left(\sum_{n=n_{1}}^{\infty} E_{n}\right)=1$, which is equivalent to (82).

Hence to prove (82) it is sufficient to prove (93). By definition this is equivalent to

$$
\sum_{\nu=1, \nu \equiv \nu_{0}(\bmod 2)}^{\infty} \operatorname{Pr}\left(\sum_{\mu=k_{\nu}^{\prime}}^{k_{v+1}^{\prime}-1} E_{\nu-1, \mu}\right)=\infty .
$$

Comparing (94) and (87) we see that in order to prove (94) it is sufficient to prove that for $\nu \neq \nu(r)$, there exists a constant $A_{10}>0$ such that for all sufficiently large $\nu$, the following shall hold:

$$
\operatorname{Pr}\left(\sum_{\mu=k_{\nu}^{\prime}}^{k_{\nu+1}^{\prime}-1} E_{v-1, \mu}\right) \geqq A_{10} \sum_{k=k_{\nu}}^{k_{\nu+1}-1} \operatorname{Pr}\left(S_{k^{\prime}}^{*}<\frac{\pi}{8^{1 / 2}} \frac{s_{k}{ }^{\prime}}{\left(\psi_{k}^{\prime 2}+8 C\right)^{1 / 2}}\right)
$$

We have for any integer $N>0$,

$$
\begin{aligned}
\operatorname{Pr}\left(\sum_{\mu=k_{\nu}^{\prime}}^{k_{v+1}^{\prime}-1} E_{\nu-1, \mu}\right) & \geqq \operatorname{Pr}\left(\sum_{k=k_{\nu}}^{k_{v+1}-1} E_{\nu-1, k^{\prime}}\right) \\
& \geqq \frac{1}{N} \sum_{k=k_{\nu}}^{k_{\nu+1}-1} \operatorname{Pr}\left(E_{\nu-1, k^{\prime}}-E_{\nu-1, k^{\prime}} \sum_{j=k+N}^{k_{v+1}} E_{\nu-1, j^{\prime}}\right) .
\end{aligned}
$$

Now we see easily that $E_{v-1, k^{\prime}} E_{v-1, j^{\prime}}$ implies $E_{k, j}^{\prime}$ where $E_{k, j}^{\prime}$ denotes the event 


$$
\max _{n_{k}<i \geqq n_{j}}\left|S_{i}-S_{n_{k}}\right|<\frac{\pi}{8^{1 / 2}}\left(\frac{s_{n_{k}}}{\left(\psi_{n_{k}}^{2}+8 C\right)^{1 / 2}}+\frac{s_{n_{j}}}{\left(\psi_{n_{j}}^{2}+8 C\right)^{1 / 2}}\right) .
$$

Since $E_{\nu-1, k^{\prime}}$ and $E_{k, j}^{\prime}$ are independent, we have

$$
\operatorname{Pr}\left(E_{\nu-1, k^{\prime}} E_{\nu-1, j^{\prime}}\right) \leqq \operatorname{Pr}\left(E_{\nu-1, k^{\prime}}\right) \operatorname{Pr}\left(E_{k, j}^{\prime}\right) .
$$

If we can prove that, for a suitable $N$,

$$
\sum_{j=k+N}^{k_{\nu+1}} \operatorname{Pr}\left(E_{k, j}^{\prime}\right)<\frac{1}{2}
$$

then from (96)

$$
\begin{aligned}
\operatorname{Pr}\left(\sum_{\mu=k_{\nu}^{\prime}}^{k_{\nu+1}^{\prime}} E_{\nu-1, \mu}\right) & \geqq \frac{1}{N} \sum_{k=k_{\nu}}^{k_{\nu+1}} \operatorname{Pr}\left(E_{\nu-1, k^{\prime}}-\sum_{j=k+N}^{k_{\nu+1}} E_{\nu-1, k^{\prime}} E_{\nu-1, j^{\prime}}\right) \\
& \geqq \frac{1}{N} \sum_{k=k_{\nu}}^{k_{\nu+1}} \operatorname{Pr}\left(E_{\nu-1, k^{\prime}}\right)\left(1-\sum_{j=k+N}^{k_{\nu+1}} \operatorname{Pr}\left(E_{k, j}^{\prime}\right)\right) \\
& \geqq \frac{1}{2 N} \cdot \sum_{k=k_{\nu}}^{k_{\nu+1}} \operatorname{Pr}\left(E_{\nu-1, k^{\prime}}\right) .
\end{aligned}
$$

By (9 bis) (4) we have

$$
\begin{aligned}
\operatorname{Pr}\left(E_{\nu-1, k^{\prime}}\right) & \leqq \operatorname{Pr}\left(\max _{k_{\nu-1}^{\prime}<\rho \leqq k^{\prime}}\left|S_{\rho}-S_{k_{\nu-1}^{\prime}}\right|<8^{-1 / 2} \pi \frac{\left(s_{k^{\prime}}^{2}-s_{k_{\nu-1}^{\prime}}^{2}\right)^{1 / 2}}{\left(\psi_{k^{\prime}}^{2}+8 C\right)^{1 / 2}}\right) \\
& \geqq \frac{A_{6}}{A_{7}} \operatorname{Pr}\left(S_{k^{\prime}}^{*}<8^{-1 / 2} \pi \frac{s_{k}{ }^{\prime}}{\left(\psi_{k^{\prime}}^{2}+8 C\right)^{1 / 2}}\right) .
\end{aligned}
$$

Thus from (98) and the last inequality we shall have proved (95) with $A_{10}=A_{6} / 2 N A_{7}$. Hence it is sufficient to prove (97).

Now we have, since $\psi_{k}^{\prime 2} \geqq \psi_{k_{\nu}}^{\prime 2} \geqq \psi_{k_{\nu+1}}^{\prime 2}-1 \geqq \psi_{j}^{\prime 2}-1$,

$$
\frac{s_{k}^{\prime}}{\left(\psi_{k}^{\prime 2}+8 C\right)^{1 / 2}}<\frac{s_{j}^{\prime}}{\left(\psi_{j}^{\prime 2}-1+8 C\right)^{1 / 2}}
$$

$$
\operatorname{Pr}\left(E_{k, j}^{\prime}\right) \leqq \operatorname{Pr}\left(\max _{n_{k} \leqq i \leqq n_{j}}\left|S_{i}-S_{n_{k}}\right|<\frac{\pi}{8^{1 / 2}}\left(s_{j}^{\prime 2}-s_{k^{\prime}}^{\prime 2}\right)^{1 / 2 \pi} g_{j}\right)
$$

where

$$
g_{j}=\frac{2 s_{j}^{\prime}}{\left(s_{j}^{\prime 2}-s_{k}^{\prime 2}\right)^{1 / 2}\left(\psi_{j}^{\prime 2}+8 C-1\right)^{1 / 2}} .
$$

(4) See footnote 3 . 
It is obvious that $g_{j} \downarrow 0$; in order to apply Theorem 2 we have to verify that

$$
\frac{\left(s_{j}^{\prime 2}-{s_{k}^{\prime}}^{2}\right)^{1 / 2}\left(\psi_{j}^{\prime 2}+8 C-1\right)^{1 / 2}}{s_{j}^{\prime}} \leqq A_{11}\left(\lg _{2}\left(s_{j}^{\prime 2}-{s_{k}^{\prime 2}}^{2}\right)\right)^{1 / 2}
$$

which is evident since

$$
\left(\frac{s_{j}^{\prime 2}-s_{k}^{\prime 2}}{\lg _{2}\left(s_{j}^{\prime 2}-s_{k}^{\prime 2}\right)}\right)^{1 / 2} \leqq A_{12} \frac{s_{j}^{\prime}}{\psi_{j}^{\prime}}
$$

by (76). Therefore we have from (99) and (9), Theorem 2 ,

$$
\operatorname{Pr}\left(E_{k, j}^{\prime}\right) \leqq A_{13} e-g_{j}^{-2}
$$

We have for sufficiently large $k$, from (77),

$$
\frac{s_{k}^{\prime}}{s_{k+1}^{\prime}} \leqq 1-\frac{a}{2 \psi_{k}^{\prime 2}}, \quad \frac{s_{k}^{\prime}}{s_{j}^{\prime}} \leqq\left(1-\frac{a}{2 \cdot \psi_{j}^{\prime 2}}\right)^{j-k} \text {. }
$$

If $h x \leqq \delta$ where $\delta>0$ is sufficiently small, then $(1-x)^{h} \leqq 1-\delta^{\prime} h x$ where $\delta^{\prime}>0$ is another constant. Hence if $j-k \leqq \delta \psi_{j}^{\prime 2}$ we have from (102)

$$
\frac{s_{k}^{\prime}}{s_{j}^{\prime}} \leqq 1-\frac{\delta^{\prime} a(j-k)}{\psi_{j}^{\prime 2}}, \quad 1-\frac{s_{k}^{\prime 2}}{s_{j}^{\prime 2}} \geqq a^{\prime} \frac{j-k}{\psi_{j}^{\prime 2}}
$$

where $a^{\prime}>0$. Then from (100)

$$
g_{j} \leqq 2\left(a^{\prime}(j-k)\right)^{-1 / 2} .
$$

Hence by (101) we have

$$
\operatorname{Pr}\left(E_{k, j}^{\prime}\right) \leqq A_{13} \exp \left(-4^{-1} a^{\prime 2}(j-k)\right) .
$$

If $h x>\delta$, then $(1-x)^{h}<\delta^{\prime \prime}<1$, hence from (102), if $j-k>\delta \psi_{j}^{\prime 2}$,

$$
\begin{gathered}
\frac{s_{k}^{\prime}}{s_{j}^{\prime}}<\delta_{0}<1, \quad 1-\frac{s_{k}^{\prime 2}}{s_{j}^{\prime 2}} \geqq 1-\delta_{0}^{2} ; \\
g_{j} \leqq 2\left(1-\delta_{0}^{2}\right)^{-1 / 2} \psi_{j}^{\prime-1} ; \\
\operatorname{Pr}\left(E_{k, j}^{\prime}\right) \leqq A_{13} \exp \left(-4^{-1}\left(1-\delta_{0}^{2}\right) \psi_{j}^{\prime 2}\right) .
\end{gathered}
$$

From (103) and (104),

(105) $\sum_{j=k+N}^{k_{\nu+1}} \operatorname{Pr}\left(E_{k, j}^{\prime}\right) \leqq A_{13}\left(\sum_{i=N}^{\infty} e^{-a^{\prime} i / 4}+\left(k_{\nu+1}-k_{\nu}\right) \exp \left(-\frac{1-\delta_{0}^{2}}{4}{\psi_{k}^{\prime 2}}^{2}\right)\right)$.

We have by (83),

$$
\psi_{k_{\nu}}^{\prime 3} \geqq \frac{s_{k_{\nu+1}}^{\prime}}{s_{k_{\nu}}^{\prime}} \geqq\left(1+\frac{a}{\psi_{k_{\nu+1}-1}^{\prime 2}}\right) \cdots\left(1+\frac{a}{\psi_{k_{\nu}}^{\prime 2}}\right) \geqq 1+\frac{a\left(k_{\nu+1}-k_{\nu}\right)}{\psi_{k_{\nu+1}}^{\prime 2}} .
$$


Hence we have

$$
k_{v+1}-k_{\nu} \leqq A_{14} \psi_{k_{\nu+1}}^{\prime 5} .
$$

Since $\nu \neq \nu(r)$, we have $\psi_{k_{\nu+1}}^{\prime 2} \leqq 2 \psi_{k \nu}^{\prime 2}$. Hence

$$
\begin{gathered}
k_{\nu+1}-k_{\nu} \leqq 6 A_{14} \psi_{k_{\nu}}^{\prime 5} \\
\left(k_{v+1}-k_{\nu}\right) \exp \left(-\frac{1-\delta_{0}^{2}}{4} \psi_{k}^{\prime 2}\right) \leqq A_{16} \psi_{k_{\nu}}^{\prime 5} e^{-A_{10} \psi_{k_{\nu}}^{\prime 2}}=o(1) .
\end{gathered}
$$

Thus by choosing $N$ sufficiently large we obtain from (105) the desired (97), if $\nu$ is sufficiently large. The proof of Lemma 9 is thus complete.

LEMMa 10. If $\left\{n_{k}\right\}, k=1,2, \cdots$, is defined by (77), then the series

$$
\sum_{k} e^{-\psi^{2} n_{k}}
$$

and

$$
\sum_{n} \frac{\sigma_{n}^{2}}{s_{n}^{2}} \psi_{n}^{2} e^{-\psi_{n}^{2}}
$$

converge and diverge together.

Proof. We have

$$
\frac{\sigma_{n}^{2}}{s_{n}^{2}}=1-\frac{s_{n-1}^{2}}{s_{n}^{2}}
$$

Since $x \leqq-\lg (1-x) \leqq 2 x$ if $0<x<1$, we obtain

$$
\begin{aligned}
& \frac{\sigma_{n}^{2}}{s_{n}^{2}} \leqq-\lg \left(1-\frac{\sigma_{n}^{2}}{s_{n}^{2}}\right)=\lg \frac{s_{n}^{2}}{s_{n-1}^{2}},
\end{aligned}
$$

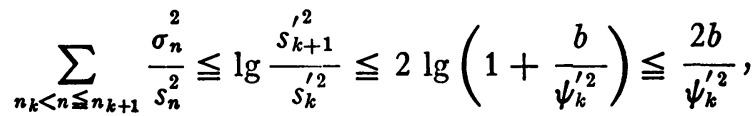

$$
\begin{aligned}
& \frac{2 \sigma_{n}^{2}}{s_{n}^{2}} \geqq-\lg \left(1-\frac{\sigma_{n}^{2}}{s_{n}^{2}}\right)=\lg \frac{s_{n}^{2}}{s_{n-1}^{2}}, \\
& 2 \sum_{n_{k}<n \leqq n_{k+1}} \frac{\sigma_{n}^{2}}{s_{n}^{2}} \geqq \lg \frac{s_{k+1}^{\prime 2}}{s_{k}^{\prime 2}} \geqq 2 \lg \left(1+\frac{a}{\psi_{k}^{\prime 2}}\right) \geqq \frac{a}{\psi_{k}^{\prime 2}} .
\end{aligned}
$$

Since $\psi_{n}^{2} e^{-\psi_{n}^{2}} \downarrow$, we have

$$
\frac{a}{2} e^{-\psi_{k+1}^{\prime 2}}=\psi_{k}^{\prime 2} e^{-\psi_{k+1}^{\prime 2}} \frac{a}{2 \psi_{k}^{\prime 2}} \leqq \sum_{n_{k}<n \leqq n_{k+1}} \frac{\sigma_{n}^{2}}{s_{n}^{2}} \psi_{n}^{2} e^{-\psi_{n}^{2}} \leqq \psi_{k}^{\prime 2} e^{-\psi_{k}^{\prime 2}} \frac{2 b}{\psi_{k}^{\prime 2}} \leqq 2 b e^{-\psi_{k}^{\prime 2}} .
$$

Lemma 10 follows from this inequality. 
Proof of Theorem 3. The $\phi_{n}$ given in (11) is monotone increasing and $\phi_{n}=O\left(\left(\lg _{2} s_{n}\right)^{1 / 2}\right)$. Hence Lemma 8 and Lemma 9 are applicable. Hence

$$
\operatorname{Pr}\left(S_{n}^{*}<8^{-1 / 2} \pi s_{n} \phi_{n}^{-1} \text { i. o. }\right)=\left\{\begin{array}{l}
0 \\
1
\end{array}\right.
$$

according as

$$
\sum_{k} e^{-\psi_{n_{k}}}\left\{\begin{array}{l}
< \\
=
\end{array}\right\} \infty .
$$

By Lemma 10, the last series converges and diverges with (13), which in this case is

$$
\sum_{n} \frac{(1+o(1)) \sigma_{n}^{2} \lg _{2} s_{n}}{s_{n}^{2} \lg s_{n}\left(\lg _{2} s_{n}\right)^{2} \lg _{3} s_{n} \cdots \lg _{p} s_{n}\left(\lg _{p+1} s_{n}\right)^{1+\delta}} .
$$

Hence a well known theorem of Abel-Dini asserts that this is convergent if and only if $\delta$ is positive. Thus Theorem 3 is proved.

Proof of Theorem 4. Suppose that $\phi_{n} \uparrow \infty$. Define

$$
\psi_{n}^{2}=\min \left(\phi_{n}^{2}, 2 \lg _{2} s_{n}\right) \text {. }
$$

If (13) is convergent, then

$$
\sum_{n} \frac{\sigma_{n}^{2}}{s_{n}^{2}} \psi_{n}^{2} e^{-\psi_{n}^{2}}=\sum_{\psi_{n}=\phi_{n}}+\sum_{\psi_{n}^{2} \geqq 21_{\mathbf{g}_{2} s_{n}}}<\infty
$$

since again by the Abel-Dini theorem we have

$$
\sum_{\psi_{n}^{2} \geqq 2 \lg _{2} s_{n}} \leqq \sum \frac{2 \sigma_{n}^{2} \lg _{2} s_{n}}{s_{n}^{2}\left(\lg s_{n}\right)^{2}}<\infty .
$$

By the definition (106) $\psi_{n}$ satisfies (76), hence by Lemma 8 ,

$$
\operatorname{Pr}\left(S_{n}^{*}<8^{-1 / 2} \pi s_{n} \psi_{n}^{-1} \text { i. o. }\right)=0 .
$$

Since $\psi_{n} \leqq \phi_{n}$, a fortiori (12) is equal to zero.

If (13) is divergent, then since $\psi_{n} \leqq \phi_{n}$, we have

$$
\sum_{n} \frac{\sigma_{n}^{2}}{s_{n}^{2}} \psi_{n}^{2} e^{-\psi_{n}^{2}}=\infty
$$

Since $\psi_{n}$ satisfies $(76)$, by Lemma $9, \operatorname{Pr}\left(S_{n}^{*}<8^{-1 / 2} \pi s_{r} \psi_{n}{ }^{-1}\right.$ i. o. $)=1$. By Theorem 3, we have

$$
\operatorname{Pr}\left(S_{n}^{*}<8^{-1 / 2} \pi \frac{s_{n}}{\left(2 \lg _{2} s_{n}\right)^{1 / 2}} \text { i. o. }\right)=0 .
$$

Hence there exists a subsequence $n_{i}$ such that $\psi_{n_{i}}^{2} \leqq 2 \lg _{2} s_{n_{i}}$ and 


$$
\operatorname{Pr}\left(S_{n_{i}}^{*}<8_{\pi s_{n_{i}}}^{-1 / 2} \psi_{n_{i}}^{-1} \text { i. o. }\right)=1 \text {. }
$$

By the definition (106), we have $\psi_{n_{i}}=\phi_{n_{i}}$. Hence (12) is equal to one. Theorem 4 is proved.

Proof of Theorem 5. By Theorem 4 it is sufficient to prove that the series

$$
\sum_{n} \frac{\sigma_{n}^{2}}{s_{n}^{2}} \phi^{2}\left(s_{n}^{2}\right) e^{-\phi^{2}\left(s_{n}^{2}\right)}
$$

and the integral (15) converge and diverge together.

We have, since $t^{-1} \phi^{2}(t) e^{-\phi^{2}(t)} \downarrow 0$,

$$
\begin{aligned}
\int_{\varepsilon_{k}^{2}}^{\infty} t^{-1} \phi^{2}(t) e^{-\phi^{2}(t)} d t & =\sum_{n=k+1}^{\infty} \int_{8_{n-1}^{2}}^{s_{n}^{2}} t^{-1} \phi^{2}(t) e^{-\phi^{2}(t)} d t \\
& \geqq \sum_{n=k+1}^{\infty} \frac{s_{n}^{2}-s_{n-1}^{2}}{s_{n}^{2}} \phi^{2}\left(s_{n}^{2}\right) e^{-\phi^{2}\left(s_{n}^{2}\right)}
\end{aligned}
$$

Hence if (107) diverges, (15) diverges too.

On the other hand, we have

$$
\sum_{n=N+1}^{\infty} \frac{\sigma_{n}^{2}}{s_{n-1}^{2}} \phi^{2}\left(s_{n-1}^{2}\right) e^{-\phi^{2}\left(s_{n-1}^{2}\right)} \geqq \int_{s_{N}^{2}}^{\infty} t^{-1} \phi^{2}(t) e^{-\phi^{2}(t)} d t .
$$

From (75) we have $s_{n}^{2}=s_{n-1}^{2}+\sigma_{n}^{2} \leqq s_{n-1}^{2}+O\left(s_{n}^{2-2 \theta}\right)$. Hence if $n$ is large enough, we have

$$
s_{n}^{2} \leqq 2 s_{n-1}^{2}
$$

Let $n_{k}, k=1,2, \cdots$, denote the subsequence of $n=1,2, \cdots$ for which

$$
\phi^{2}\left(s_{n_{k}}^{2}\right)>\phi^{2}\left(s_{n_{k}-1}^{2}\right)+1 .
$$

Evidently we have by (109) and (110),

$$
\sum_{k} \frac{\sigma_{n_{k}}^{2}}{s_{n_{k}-1}^{2}} \phi^{2}\left(s_{n_{k}-1}^{2}\right) e^{-\phi^{2}\left(s_{n_{k}-1}^{2}\right)} \leqq A_{16} \sum_{k} \frac{\sigma_{n_{k}}^{2}}{s_{n_{k}}^{2}} e^{-\phi^{2}\left(8_{n_{k}}^{2}-1\right) / 2}<\infty .
$$

Hence if (15) diverges, we have, by (108) and (111),

$$
\sum_{n=1, n \neq n_{k}}^{\infty} \frac{\sigma_{n}^{2}}{s_{n-1}^{2}} \phi^{2}\left(s_{n-1}^{2}\right) e^{-\phi^{2}\left(s_{n-1}^{2}\right)}=\infty .
$$

By (110) if $n \neq n_{k}$, we have $\phi^{2}\left(s_{n}^{2}\right) \leqq \phi^{2}\left(s_{n-1}^{2}\right)+1$. From this and (112) we obtain

$$
\sum \frac{\sigma_{n}^{2}}{s_{n}^{2}} \phi^{2}\left(s_{n}^{2}\right) e^{-\phi^{2}\left(s_{n}^{2}\right)} \geqq \frac{e^{-1}}{2} \sum \frac{\sigma_{n}^{2}}{s_{n-1}^{2}} \phi^{2}\left(s_{n-1}^{2}\right) e^{-\phi^{2}\left(s_{n-1}^{2}\right)}=\infty .
$$

Theorem 5 is proved. 


\section{REFERENCES}

1. L. Bachelier, Calcul des probabilités, vol. 1, Paris, 1912.

2. H. Bergström, On the central limit theorem in $R_{k}, k>1$, Skand. Aktuarietidskr. vol. 28 (1945) pp. 106-127.

3. A. C. Berry, The accuracy of the Gaussian approximation to the sum of independent variates, Trans. Amer. Math. Soc. vol. 49 (1941) pp. 122-136.

4. K. L. Chung and Paul Erdös, On the lower limit of sums of independent random variables, Ann. of Math. vol. 48 (1947) pp. 1003-1013.

5. H. Cramér, Random variables and probability distributions, Cambridge Tracts in Mathematics, No. 36, Cambridge University Press, 1937.

6.- Sur un nouveau théorème-limite de la théorie des probabiłitếs, Actualités Scientifiques, No. 736, Paris, 1938, pp. 5-23. 1946.)

7. - Mathematical methods of statistics, Uppsala, 1945. (Princeton University Press,

8. P. Erdös and M. Kac, On certain limit theorems of the theory of probability, Bull. Amer. Math. Soc. vol. 52 (1946) pp. 292-302.

9. C.-G. Esseen, Fourier analysis of distribution functions, Acta Math. vol. 77 (1945) pp. $1-125$.

10. W. Feller, Generalization of a probability limit theorem of Cramér, Trans. Amer. Math. Soc. vol. 54 (1943) pp. 373-402.

11. - The general form of the so-called law of the iterated logarithm, Trans. Amer. Math. Soc. vol. 54 (1943) pp. 373-402.

12. - The fundamental limit theorems in probability, Bull. Amer. Math. Soc. vol. 51 (1945) pp. 800-832.

13. M. Fréchet, Recherches théoriques modernes sur la théorie des probabilités, vol. 1, Paris, 1937.

14. A. Khintchine, Ein Satz der Wahrscheinlichkeitsrechnung, Fund. Math. vol. 6 (1924) pp. 9-10.

15. A. Kolmogoroff, Ueber die Summen durch den Zufall bestimmter unabhängiger Grössen, Math. Ann. vol. 99 (1928) pp. 309-319.

16. - - Ueber das Gesetz des iterierten Logarithmus, Math. Ann. vol. 101 (1929) pp. 126-135.

17. A. Liapounoff, Nouvelle forme du théorème sur la limite de la probabilité, Mémoires de l'Académie de Saint-Petersbourg (8) vol. 12 (1901).

18. J. V. Uspensky, Introduction to mathematical probability, New York, McGraw-Hill, 1937.

Princeton University,

Princeton, N. J. 